\title{
Perfil de ácidos graxos e estabilidade oxidativa do leite de vacas holandesas alimentadas com soja extrusada e selênio orgânico
}

\author{
Juliana Jorge Paschoal(1), Marcus Antonio Zanetti(1), Gustavo Ribeiro Del Claro(1), Mariza Pires de Melo(1), \\ Silvana Piccoli Pugine ${ }^{(1)}$ e José Aparecido Cunha ${ }^{(1)}$
}

(1)Universidade de São Paulo, Fac. de Zootecnia e Engenharia de Alimentos, Av. Duque de Caxias Norte, no 225, CEP 13600-000 Pirassununga, SP. E-mail: julianajp@yahoo.com.br, mzanetti@usp.br, gdelclaro@yahoo.com.br, mpmelo@usp.br, spiccoli@usp.br, jcunha@usp.br

\begin{abstract}
Resumo - O objetivo deste trabalho foi avaliar o efeito de dieta com alto teor de soja extrusada e selênio orgânico sobre o perfil de ácidos graxos e a estabilidade oxidativa do leite de vacas da raça Holandesa. Vinte e quatro vacas foram divididas em três blocos e distribuídas ao acaso em três tratamentos: dieta controle; dieta com $21 \%$ de soja extrusada e dieta com $21 \%$ de soja extrusada $+5 \mathrm{mg}$ de selênio orgânico. O experimento teve duração de seis semanas. As vacas alimentadas com soja extrusada produziram leite com menor concentração de ácidos graxos de cadeias curta e média, maior concentração de ácidos graxos de cadeia longa, menor concentração de ácidos graxos saturados e maior concentração de ácidos graxos poliinsaturados e de ácido linoléico conjugado. A suplementação com selênio aumentou a sua concentração no leite e retardou o processo oxidativo do leite. A dieta com $21 \%$ de soja extrusada alterou o perfil de ácidos graxos do leite, aumentando sua susceptibilidade à oxidação; o enriquecimento com selênio minimizou esse efeito e influenciou positivamente a estabilidade do leite.
\end{abstract}

Termos para indexação: antioxidante, ácido linoléico conjugado, índice de peróxidos, TBARS.

\section{Fatty acid profile and oxidative stability of milk from Holstein cows fed with extruded soybean and organic selenium}

\begin{abstract}
The objective of this work was to evaluate the effect of extruded soybean and organic selenium supplementation on fatty acid profile and oxidative stability of Holstein milk. Twenty-four Holstein cows were distributed in three blocks and were allocated in three treatments: control diet; diet with $21 \%$ extruded soybean; and diet with $21 \%$ of extruded soybean $+5 \mathrm{mg}$ of organic selenium. The experiment lasted six weeks. The cows fed with extruded soybean diet produced milk with lower concentration of short and medium chain fatty acids and higher concentration of long chain fatty acids, lower concentration of saturated fatty acids and higher concentration of polyunsaturated fatty acids and conjugated linoleic acid content. The organic selenium supplementation increased milk selenium concentration and delayed milk oxidation. Diet with $21 \%$ extruded soybean modified fatty acid profile and resulted in milk susceptible to oxidation. Organic selenium supplementation reduced oxidation and influenced positively the stability of milk.
\end{abstract}

Index terms: antioxidant, conjugated linoleic acid, peroxide index, TBARS.

\section{Introdução}

O leite bovino é composto por mais de 100 mil tipos diferentes de moléculas (cada uma delas com função específica), contém de 3 a 5\% de lipídios totais, representados em quase sua totalidade por triacilgliceróis encontrados nos glóbulos de gordura (Fonseca \& Santos, 2000). Cerca de $70 \%$ dos ácidos graxos dos triacilgliceróis do leite são saturados, $25 \%$ são ácidos graxos monoinsaturados e 5\% são ácidos graxos poliinsaturados (Grummer, 1991).
A tendência atual é a demanda crescente por alimentos saudáveis, com baixos teores de gordura saturada e, preferencialmente, com fatores que atuem na promoção de efeitos fisiológicos benéficos à saúde (Padilha \& Pinheiro, 2004).

O fornecimento aos animais de dietas com alta inclusão de soja extrusada pode alterar a concentração e a composição da gordura do leite, pela inibição da bio-hidrogenação e o conseqüente aumento da passagem de ácidos graxos poliinsaturados pelo rúmen, o que aumenta a absorção intestinal e a incorporação ao leite 
(Beam et al., 2000). Além disso, a bio-hidrogenação incompleta do ácido graxo linoléico, presente em grande quantidade na soja extrusada, pode aumentar a concentração de ácido linoléico conjugado (CLA) no leite (AbuGhazaleh et al., 2002), considerado um importante agente anticarcinogênico natural (Ip et al., 1999; Lock et al., 2004).

Dhiman et al. (1999), Abughazaleh et al. (2002a) e Kristensen et al. (2004) constataram diminuição na concentração de ácidos graxos saturados e aumento na concentração de ácidos graxos poliinsaturados e CLA no leite de vacas alimentadas com dieta com alta inclusão de soja extrusada. De acordo com Ramaswamy et al. (2001), a dieta com soja extrusada aumentou a concentração de ácidos graxos poliinsaturados e CLA no leite, creme de leite, queijo e manteiga, quando comparada à dieta controle. Esta modificação no perfil de ácidos graxos pode alterar várias propriedades físicas e químicas da gordura do leite, como firmeza, ponto de fusão, viscosidade, estabilidade oxidativa e "flavor" (Kaylegian \& Lindsay, 1995). Timmons et al. (2001) observaram correlação positiva $(0,82)$ entre a porcentagem de soja tostada na dieta e a concentração de ácidos graxos poliinsaturados no leite. O desenvolvimento de "flavor" oxidado após oito dias de armazenamento foi correlacionado positivamente à concentração de ácidos graxos poliinsaturados totais $(0,50)$. Os lipídios são protegidos contra a oxidação por antioxidantes naturais presentes no leite in natura (Manson \& Akesson, 2000). Entre as enzimas antioxidantes, a glutationa peroxidase, que é Se-dependente, age sobre os hidroperóxidos, compostos primários da oxidação lipídica (Surai, 2006). No decurso da decomposição dos hidroperóxidos, ocorre formação dos chamados produtos secundários da oxidação (aldeídos, cetonas, hidroxiácidos, hidrocarbonetos, polímeros), e vários desses compostos são voláteis e responsáveis pela formação de "off-flavor" (Manson \& Akesson, 2000). A atividade da enzima depende da biodisponibilidade do mineral que, por sua vez, é dependente da fonte utilizada (Surai, 2006). Muñiz-Naviero et al. (2006) avaliaram a concentração de Se no leite e observaram maior biodisponibilidade do mineral no tratamento com Se orgânico, comparado ao tratamento com selenito de sódio. Nicholson et al. (1991) estudaram a administração de duas fontes de $\mathrm{Se}$ (orgânico e inorgânico), associadas ou não à vitamina $\mathrm{E}$ (3.000 UI por dia), em seu efeito sobre a estabilidade oxidativa do leite avaliada por análise sensorial. Os autores observaram maior concentração de Se no leite, no tratamento com Se orgânico, e concluíram que a concentração adequada de $\mathrm{Se}$ aumentou a transferência de vitamina E para o leite. Não foi encontrado efeito dos tratamentos sobre o desenvolvimento de "flavor" oxidado.

O objetivo deste trabalho foi avaliar o efeito da dieta com alto teor de soja extrusada e Se orgânico sobre o perfil de ácidos graxos, a concentração de ácido linoléico conjugado, a concentração de $\mathrm{Se}$ e a estabilidade oxidativa do leite de vacas da raça Holandesa.

\section{Material e Métodos}

O experimento foi realizado no setor leiteiro da Prefeitura do Campus Administrativo da USP, em Pirassununga, SP, no primeiro semestre de 2005. Foram utilizadas 24 vacas leiteiras da raça Holandesa, de 60 a 240 dias em lactação, com produção média de $23 \mathrm{~kg}$ de leite por dia. A ordenha foi realizada duas vezes ao dia (6 e 15h) em sala de ordenha do tipo espinha-de-peixe. As medidas gerais de higiene consistiram em lavagem dos tetos, secagem com papel toalha, pré e pós "dipping" com antisséptico. Entre as ordenhas, os animais foram alojados em instalação do tipo "free-stall" com cochos coletivos.

Os animais foram divididos em três blocos, de acordo com o número de lactações: bloco 1 - seis novilhas; bloco 2 - 12 vacas de duas a cinco lactações; bloco 3 seis vacas, com mais de cinco lactações, distribuídas ao acaso em três tratamentos, cada um composto por duas novilhas, quatro vacas de duas a cinco lactações e duas vacas com mais de cinco lactações. Os tratamentos foram: dieta controle (controle), dieta com $21 \%$ de soja extrusada (SojaExt) e dieta com $21 \%$ de soja extrusada $+5 \mathrm{mg}$ de Se orgânico (SojaExt+Se).

O Se orgânico utilizado (Zimpro) continha 1.000 ppm de Se, na forma de selênio-metionina. A dose diária ( $5 \mathrm{mg}$ ) foi pesada, acondicionada em saquinhos de papel $(13,5 \times 7 \mathrm{~cm})$ imersos em melaço de cana, antes de serem colocados dentro da boca dos animais, para garantir consumo total.

As vacas foram alimentadas com dieta total de $56 \%$ de volumoso ( $89 \%$ de silagem de milho e $11 \%$ de feno de coast-cross) e $44 \%$ de concentrado, na matéria seca. As dietas foram balanceadas para manutenção dos mesmos níveis de energia e proteína (Tabela 1). 
O período de adaptação ao manejo e à dieta foi de 14 dias, e o período experimental teve duração de seis semanas. Amostras dos concentrados, feno, silagem e das sobras foram colhidas, semanalmente, para análises de: matéria seca (MS), proteína bruta (PB), extrato etéreo $(\mathrm{EE})$, matéria mineral $(\mathrm{MM})$, cálcio $(\mathrm{Ca})$ e fósforo $(\mathrm{P})$ (Association of Official Analytical Chemists, 1990); fibra em detergente neutro (FDN) (Silva \& Queiroz, 2002); e Se (Olson et al., 1975). Os nutrientes digestíveis totais (NDT) foram determinados conforme Kearl (1982).

No levantamento do perfil de ácidos graxos, as amostras de leite foram colhidas no último dia experimental, durante a ordenha da manhã, ao final da extração total do leite dos animais, diretamente do balão volumétrico, após $5 \mathrm{~s}$ de homogeneização. Foram utilizados tubos de plástico, com capacidade para $100 \mathrm{~mL}$, sem adição de conservantes, e as amostras foram congeladas a $-20^{\circ} \mathrm{C}$.

$\mathrm{O}$ perfil de ácidos graxos foi determinado no Laboratório de Nutrição e Crescimento Animal, da

Tabela 1. Ingredientes e composição química das dietas experimentais (valores expressos em porcentagem da matéria seca $)^{(1)}$.

\begin{tabular}{|c|c|c|c|}
\hline Ingrediente & Controle & SojaExt & SojaExt+Se \\
\hline Silagem de milho & 50,00 & 50,00 & 50,00 \\
\hline Feno de coast-cross & 6,00 & 6,00 & 6,00 \\
\hline Fubá de milho & - & 10,47 & 10,47 \\
\hline Milho moído & 26,40 & - & - \\
\hline Casca de soja & - & 6,952 & 6,952 \\
\hline Farelo de soja & 8,80 & - & - \\
\hline Soja extrusada & - & 20,86 & 20,86 \\
\hline Farinha de peixe & 1,98 & 2,99 & 2,99 \\
\hline Farelo de trigo & 3,52 & - & - \\
\hline Uréia & 0,89 & 0,32 & 0,32 \\
\hline Calcáreo & 0,26 & - & - \\
\hline Óxido de magnésio & 0,26 & 0,26 & 0,26 \\
\hline Sal comum & 0,44 & 0,44 & 0,44 \\
\hline Premix mineral $^{(1)}$ & 0,88 & 0,88 & 0,88 \\
\hline Sulfato de amônia & 0,04 & 0,04 & 0,04 \\
\hline Bicarbonato de sódio & 0,53 & 0,53 & 0,53 \\
\hline \multicolumn{4}{|l|}{ Composição química } \\
\hline Matéria seca $(\%)$ & 54,20 & 54,90 & 54,90 \\
\hline Proteína bruta $(\%)^{(2)}$ & 17,10 & 17,30 & 17,30 \\
\hline Extrato etéreo (\%) & 3,40 & 7,10 & 7,10 \\
\hline Fibra detergente neutro $(\%)$ & 36,70 & 39,50 & 39,50 \\
\hline Matéria mineral (\%) & 5,90 & 6,10 & 6,10 \\
\hline Nutrientes digestíveis totais (\%) & 71,50 & 72,00 & 72,00 \\
\hline Selênio (ppm) & 0,31 & 0,31 & 0,54 \\
\hline
\end{tabular}

${ }^{(1)}$ Composição por quilograma do produto: S, $80 \mathrm{~g} ; \mathrm{Mg}, 20 \mathrm{~g} ; \mathrm{K}, 20 \mathrm{~g}$; $\mathrm{Mn}, 1.000 \mathrm{mg}$; Zn, $2.500 \mathrm{mg}$; Cu, $1.500 \mathrm{mg}$; Co, $100 \mathrm{mg}$; I, $80 \mathrm{mg}$; Se, 20 mg; Ca, 180 g; P, 90 g; F máx., 300 mg. (2)Os teores de proteína bruta foram utilizados para a formulação de uma dieta isoprotéica e isoenergética à utilizada pelo rebanho.
Escola Superior de Agricultura Luiz de Queiroz. $\mathrm{Na}$ extração da gordura, as amostras de leite foram descongeladas e centrifugadas (11.000 rpm) por $30 \mathrm{~min}$. Do sobrenadante, foram retiradas $400 \mathrm{mg}$ para a extração da gordura (Hara \& Radim, 1978). A metilação foi feita por meio do uso de solução metanólica de metóxido de sódio (Christie, 1982). O perfil de ácidos graxos foi determinado por cromatografia gasosa (ThermoFinnigan, modelo Trace 2000), com coluna capilar de sílica fundida SP-2560 (100x0,25x0,2 mm) e detector de ionização de chama. $\mathrm{O}$ hidrogênio foi utilizado como gás de arraste a $1 \mathrm{~mL}$ por min, e as temperaturas do injetor e detector foram de 250 e $300^{\circ} \mathrm{C}$, respectivamente. A razão de injeção das amostras foi de 25:1, o fluxo de ar foi ajustado a $460 \mathrm{~mL}$ por min, e o fluxo de nitrogênio (gás auxiliar) a $30 \mathrm{~mL}$ por min. A temperatura inicial foi de $70^{\circ} \mathrm{C}$. Quatro minutos após a injeção das amostras, a temperatura foi elevada a $175^{\circ} \mathrm{C}\left(13^{\circ} \mathrm{C}\right.$ por min) e, então, mantida por $27 \mathrm{~min}$. Por fim, a temperatura foi elevada a $215^{\circ} \mathrm{C}\left(4^{\circ} \mathrm{C}\right.$ por min) e mantida por $21 \mathrm{~min}$. Um padrão de manteiga (CRM-164; Commisssion of the European Communities, Comunity Bureau of Reference, Brussels, Belgium), com os valores certificados para alguns ácidos graxos, especialmente os de cadeia curta, foi utilizado para determinar suas recuperações e calcular os respectivos fatores de correção. A concentração dos ácidos graxos foi expressada em gramas por $100 \mathrm{~g}$ de ácidos graxos totais.

No acompanhamento dos produtos iniciais da oxidação, $500 \mathrm{~mL}$ de leite foram colhidos no último dia do experimento, em recipientes de plástico, durante a ordenha da manhã, diretamente do balão volumétrico, após $5 \mathrm{~s}$ de homogeneização. As amostras foram imediatamente levadas ao laboratório para extração da gordura (Feng et al., 2004), pelo método de centrifugação. Sessenta mililitros de leite fresco foram centrifugados a $17.800 \mathrm{~g}$ por $30 \mathrm{~min} \mathrm{a} 4^{\circ} \mathrm{C}$. Uma alíquota de $1 \mathrm{~g}$ do sobrenadante foi transferida para um microtubo e novamente centrifugada à temperatura ambiente por $20 \mathrm{~min}$, à rotação de $19.300 \mathrm{~g}$. Finalmente, $100 \mu \mathrm{L}$ foram retirados da camada superficial, para determinação do índice de peróxido (International Dairy Federation, 1991). Cada amostra $(100 \mu \mathrm{L})$ foi colocada em tubo de vidro com 9,8 mL de solução clorofórmio-metanol (7+3 V/V) sob constante agitação. Foram adicionados $50 \mu \mathrm{L}$ de solução de tiocianato de amônio $+50 \mu \mathrm{L}$ de solução de $\mathrm{Fe}^{2+}$. Após 5 min de incubação à temperatura ambiente, 
a absorbância foi determinada a $500 \mathrm{~nm}$ em espectrofotômetro, contra o branco (solução com todos os reagentes menos a amostra). De acordo com esse método, os peróxidos presentes na gordura oxidam o $\mathrm{Fe}^{2+} \mathrm{a} \mathrm{Fe}^{3+}$, e as leituras são realizadas em comprimento de onda de $500 \mathrm{~nm}$, sob a forma de tiocianato férrico. Os mesmos procedimentos (extração e análise de peróxido) foram repetidos nas mesmas amostras, $24 \mathrm{e}$ 96 horas após a colheita.

Nos produtos secundários de oxidação, análises de reações com ácido tiobarbitúrico (TBARS) foram realizadas imediatamente após a colheita de leite, e 24 e 96 horas depois da colheita (King, 1962), e 17,6 mL de leite foram colocados em frasco com tampa de vidro e aquecidos a $30^{\circ} \mathrm{C}$. Foram adicionados: $1 \mathrm{~mL}$ de solução de ácido tricloroacético $\left(1 \mathrm{~g} \mathrm{~mL}^{-1}\right)$ e $2 \mathrm{~mL}$ de etanol $95 \%$, sob agitação vagarosa. Após $5 \mathrm{~min}$ em repouso, a solução foi filtrada em papel Whatman no 42 , e $4 \mathrm{~mL}$ do filtrado foram retirados e misturados a $1 \mathrm{~mL}$ de solução de ácido 2-tiobarbitúrico (1,4 g de ácido 2-tiobarbitúrico, dissolvido em $100 \mathrm{~mL}$ de etanol 95\%). A nova solução foi colocada em banho-maria por $60 \mathrm{~min}$ a $60^{\circ} \mathrm{C}$ e, após resfriamento completo, a leitura foi realizada em espectrofotômetro a $532 \mathrm{~nm}$, com água destilada como padrão. O teste do ácido 2-tiobarbitúrico baseia-se na reação do ácido tiobarbitúrico com os produtos de decomposição dos hidroperóxidos. Um dos principais produtos formados no processo oxidativo é o malonaldeído, que é um aldeído com três átomos de carbono. Geralmente, uma molécula de malonaldeído reage com duas moléculas de ácido 2-tiobarbitúrico, para formar um complexo de cor vermelha, o qual absorve a $532 \mathrm{~nm}$.

Os índices de peróxido e TBARS foram comparados em cada tempo de colheita pelo procedimento Proc Mixed do SAS (SAS Institute, 2000), e os dados de perfil de ácidos graxos foram analisados pelo PROC GLM do SAS (SAS Institute, 2000).

\section{Resultados e Discussão}

Os valores obtidos do perfil de ácidos graxos das amostras de leite (Tabela 2) foram semelhantes aos encontrados por Ramaswamy et al. (2001), em vacas alimentadas com forragem conservada e concentrado com alta concentração de óleo vegetal.

O leite das vacas alimentadas com soja extrusada (SojaExt e SojaExt+Se) apresentou menor concentração de ácidos graxos de cadeias curta e média $(\mathrm{p}<0,01)$ e maior concentração de ácidos graxos de cadeia longa $(\mathrm{p}<0,01)$. Dhiman et al. (1999) e Abughazaleh et al. (2002a) também encontraram aumento na concentração de ácidos graxos de cadeia longa, quando forneceram dieta com alta inclusão de soja extrusada às vacas leiteiras.

Resultados satisfatórios foram observados em relação ao teor de saturação dos ácidos graxos. O leite das vacas alimentadas com soja extrusada (SojaExt e SojaExt+Se) apresentou menor concentração de ácidos graxos saturados e maior concentração de ácidos graxos poliinsaturados e monoinsaturados, quando comparado ao grupo controle (Tabela 2). O mesmo resultado foi verificado por Dhiman et al. (1999), Abughazaleh et al. (2002a) e Kristensen et al. (2004).

No presente trabalho, o isômero biologicamente ativo (cis-9 trans-11) aumentou 3,2 vezes no leite das vacas alimentadas com soja extrusada ( $\mathrm{p}<0,01)$. Essa tendência foi observada por Ramaswamy et al. (2001) e Abughazaleh et al. (2002), porém, os valores encontrados por esses autores foram mais altos, 1,24 e $0,87 \mathrm{~g}$ por $100 \mathrm{~g}$, respectivamente, contra $0,29 \mathrm{~g}$ por $100 \mathrm{~g}$ obtido neste trabalho. Em estudo desenvolvido no Brasil, Santos et al. (2001) encontraram concentração

Tabela 2. Perfil de ácidos graxos do leite (g por $100 \mathrm{~g}$ ) de vacas que receberam dieta controle, dieta com $21 \%$ de soja extrusada (SojaExt) e dieta com $21 \%$ de soja extrusada $+5 \mathrm{mg}$ de selênio $(\text { SojaExt+Se })^{(1)}$.

\begin{tabular}{|c|c|c|c|c|c|c|}
\hline Perfil & Controle & SojaExt & SojaExt+Se & DP & $\mathrm{CV}(\%)$ & Trat \\
\hline $\mathrm{C} 4: 0$ & $4,34 \mathrm{a}$ & $3,40 \mathrm{~b}$ & $3,61 \mathrm{~b}$ & 0,670 & 17,7 & $*$ \\
\hline C6:0 & $2,15 \mathrm{a}$ & $1,31 \mathrm{~b}$ & $1,35 \mathrm{~b}$ & 0,250 & 15,5 & $* *$ \\
\hline $\mathrm{C} 8: 0$ & $1,19 \mathrm{a}$ & $0,61 b$ & $0,64 b$ & 0,146 & 17,9 & $* *$ \\
\hline C10:0 & $2,57 \mathrm{a}$ & $1,56 \mathrm{~b}$ & $1,30 \mathrm{~b}$ & 0,426 & 23,5 & $* *$ \\
\hline C12:0 & $2,83 \mathrm{a}$ & $1,48 \mathrm{~b}$ & $1,57 \mathrm{~b}$ & 0,461 & 23,5 & $* *$ \\
\hline $\mathrm{C} 14: 0$ & $9,32 \mathrm{a}$ & $6,43 b$ & $6,10 \mathrm{~b}$ & 1,328 & 18,2 & $* *$ \\
\hline $\mathrm{C} 16: 0$ & $26,79 a$ & $21,54 b$ & $20,41 b$ & 1,966 & 8,5 & $* *$ \\
\hline C18:0 & $14,88 \mathrm{~b}$ & $18,97 \mathrm{a}$ & $19,14 \mathrm{a}$ & 3,334 & 18,0 & $*$ \\
\hline C18:1 & $28,96 \mathrm{~b}$ & $35,72 \mathrm{a}$ & $36,73 a$ & 3,532 & 10,4 & $* *$ \\
\hline C18:1 trans 11 & $1,29 b$ & $5,15 \mathrm{a}$ & $3,53 \mathrm{a}$ & 0,959 & 28,8 & $* *$ \\
\hline C18:2 & $2,46 b$ & $3,79 \mathrm{a}$ & $3,70 \mathrm{a}$ & 0,423 & 12,7 & $* *$ \\
\hline $\mathrm{C} 18: 2 \operatorname{cis} 9 \operatorname{trans} 11$ & $0,09 \mathrm{~b}$ & $0,29 \mathrm{a}$ & $0,28 \mathrm{a}$ & 0,058 & 26,4 & $* *$ \\
\hline C18:3 & $0,68 \mathrm{a}$ & $0,76 \mathrm{a}$ & $0,76 a$ & 0,159 & 21,6 & $\mathrm{~ns}$ \\
\hline $\mathrm{C} 20-24$ & $0,63 \mathrm{a}$ & $0,71 \mathrm{a}$ & $0,70 \mathrm{a}$ & 0,080 & 11,7 & $\mathrm{~ns}$ \\
\hline Cadeia curta (C4-C12) & $13,24 \mathrm{a}$ & $8,13 b$ & $8,56 b$ & 1,366 & 13,6 & $* *$ \\
\hline Cadeia média (C13-C17) & $41,00 \mathrm{a}$ & $31,82 b$ & $30,22 b$ & 3,198 & 9,3 & $* *$ \\
\hline Cadeia longa (C18) & $45,16 b$ & $59,39 a$ & $60,52 \mathrm{a}$ & 3,978 & 7,2 & $* *$ \\
\hline Saturados & $64,00 \mathrm{a}$ & $55,3 \mathrm{~b}$ & $54,12 \mathrm{~b}$ & 3,880 & 9,6 & $* *$ \\
\hline Monoinsaturados & $28,96 b$ & $35,72 \mathrm{a}$ & $36,73 a$ & 3,529 & 10,4 & $* *$ \\
\hline Poliinsaturados & $3,15 b$ & $4,55 \mathrm{a}$ & $4,46 \mathrm{a}$ & 0,366 & 9,3 & $* *$ \\
\hline
\end{tabular}

Pesq. agropec. bras., Brasília, v.42, n.12, p.1793-1799, dez. 2007 
de $0,18 \mathrm{~g}$ de CLA por $100 \mathrm{~g}$ de gordura, no leite de vacas alimentadas com grão de soja triturado. Os autores afirmaram que o baixo nível de CLA encontrado poderia estar relacionado à baixa disponibilidade dos lipídios à bio-hidrogenação, uma vez que estariam presos à matriz protéica da semente.

A concentração de ácido graxo C18:1 trans-11 (transvacênico - TVA) aumentou no leite das vacas que receberam as dietas com soja extrusada (Tabela 2). $\mathrm{O}$ aumento na concentração de TVA é benéfico, pois os tecidos podem sintetizar CLA (cis-9 trans-11) a partir do TVA, pela ação da enzima delta-9 dessaturase. De acordo com Corl et al. (2000), a delta-9 dessaturase é responsável pela produção de $65 \%$ do isômero de CLA cis-9, trans-11 na gordura do leite. Os valores de TVA, encontrados neste trabalho, foram mais elevados que os observados por Ramaswamy et al. (2001), ao contrário dos teores de CLA. Essa discrepância de valores pode estar relacionada à ação ineficiente da enzima, que compromete os valores de CLA.

$\mathrm{O}$ teor médio do Se na dieta basal, durante o período experimental, foi $0,31 \mathrm{mg} \mathrm{kg}^{-1}$ (0,31 ppm), oque representou consumo de 6,5 mg de Se por dia. As vacas, suplementadas com $5 \mathrm{mg}$ de Se por dia, passaram a consumir, portanto, 11,52 mg de Se diário ou 0,54 $\mathrm{mg} \mathrm{kg}^{-1} \mathrm{MS}(0,54 \mathrm{ppm})$. Os níveis séricos de Se não apresentaram diferenças significativas em razão dos tratamentos (Tabela 3). Esses resultados não eram esperados, e uma possível explicação poderia estar relacionada à lise das células vermelhas, que ocorre no processo de remoção do coágulo durante o procedimento de pipetagem do soro. O ciclo de vida das células vermelhas é de 90 a 120 dias, e, portanto, o período do experimento pode não ter sido suficiente para que houvesse completo turnover de células vermelhas em resposta à suplementação com Se. Dessa forma, a lise das células vermelhas pode ter afetado a concentração de Se no soro (Surai, 2006).

A concentração de $\mathrm{Se}$, no leite das vacas suplementadas com o mineral, foi maior $(\mathrm{p}<0,05)$ quando comparada aos dos outros dois tratamentos (Tabela 3 ). Também foi verificado efeito de tempo para essa variável. Antes do início dos tratamentos (tempo 0), os níveis de Se foram semelhantes $(0,02561,0,02650$, $0,02336 \mathrm{mg} \mathrm{mL}^{-1}$, para os tratamentos controle, soja extrusada e soja extrusada $+\mathrm{Se}$, respectivamente). A partir da terceira semana de suplementação, as vacas que receberam Se orgânico produziram leite com maior teor de Se $(0,0272,0,0267,0,0312$ para os tratamentos controle, soja extrusada e soja extrusada $+\mathrm{Se}$, respectivamente). Esse resultado indica que a concentração e o período de suplementação foram suficientes. Resultado bastante próximo foi obtido por Muñiz-Naviero et al. (2006), quando suplementaram vacas com $0,5 \mathrm{ppm}$ de Se orgânico $\left(0,020 \mu \mathrm{g} \mathrm{mL}^{-1} \mathrm{e}\right.$ $0,039 \mu \mathrm{g} \mathrm{mL}^{-1}$ para os tratamentos controle e Se, respectivamente).

Segundo Labuza (1975), os níveis de peróxido aumentam vagarosamente nos estágios iniciais de oxidação, mas ao final do período de indução, a concentração de peróxido aumenta rapidamente e, então, declina e alcança valores próximos de zero. Foram observados altos valores de peróxido, nas primeiras sete horas pós-colheita, o que indica grande concentração de hidroperóxidos e término da fase de indução (Figura 1). Foi observada queda acentuada após 32 horas de armazenamento, e manutenção dos índices de peróxido 102 horas após a colheita do leite. Os índices de peróxido obtidos não estão de acordo com a clássica curva da formação dos hidroperóxidos e subseqüente quebra em inúmeros produtos de reação. Provavelmente, a formação dos hidroperóxidos foi iniciada durante a colheita e processamento das amostras, antes das primeiras sete horas.

O leite com perfil modificado de ácidos graxos, oriundo das vacas alimentadas com alta inclusão de soja extrusada (SojaExt), apresentou maior índice de

Tabela 3. Concentração de selênio $\left(\mu \mathrm{g} \mathrm{mL} \mathrm{m}^{-1}\right)$ no soro sangüíneo e no leite, índice de peróxido (meq de $\mathrm{O}_{2} \mathrm{~kg}^{-1}$ de óleo) e valores de análises de reações com ácido tiobarbitúrico (TBARS) (absorbância $532 \mathrm{~nm}$ ) no leite de vacas que receberam dieta controle, dieta com $21 \%$ de soja extrusada (sojaExt) e dieta com $21 \%$ de soja extrusada $+5 \mathrm{mg}$ de selênio $(\text { sojaExt+Se) })^{(1)}$.

\begin{tabular}{lcccc}
\hline Tratamento & $\begin{array}{c}\text { Selênio } \\
\text { no soro }\end{array}$ & $\begin{array}{c}\text { Selênio } \\
\text { no leite }\end{array}$ & $\begin{array}{c}\text { Índice de } \\
\text { peróxido }\end{array}$ & TBARS \\
\hline Controle & $0,0789 \mathrm{a}$ & $0,0272 \mathrm{~b}$ & $0,2469 \mathrm{~b}$ & $0,0607 \mathrm{a}$ \\
SojaExt & $0,0791 \mathrm{a}$ & $0,0267 \mathrm{~b}$ & $0,3399 \mathrm{a}$ & $0,0553 \mathrm{a}$ \\
SojaExt+Se & $0,0769 \mathrm{a}$ & $0,0312 \mathrm{a}$ & $0,2340 \mathrm{~b}$ & $0,0341 \mathrm{a}$ \\
\hline DP & 0,0027 & 0,0012 & 0,0271 & 0,0124 \\
CV $(\%)$ & 3,44 & 4,22 & 9,9 & 24,8 \\
\hline Trat & $\mathrm{ns}$ & $*$ & $*$ & $\mathrm{~ns}$ \\
Tempo & $* *$ & $* *$ & $* *$ & $* *$ \\
\hline
\end{tabular}

${ }^{(1)}$ Médias seguidas pela mesma letra, na coluna, não diferem entre si pelo

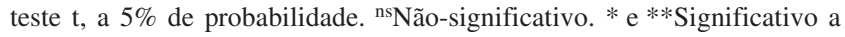
5 e $1 \%$ de probabilidade, respectivamente, pelo teste $\mathrm{F}$. 
peróxido, quando comparado ao leite das vacas dos tratamentos-controle e SojaExt+Se (Tabela 3), o que comprova que a suplementação com Se, realizada no último tratamento, influenciou positivamente a oxidação do leite com perfil modificado de ácidos graxos. Os valores obtidos neste trabalho estão próximos do valor padrão especificado pelo International Dairy Federation (1991) de 0,2 meq de $\mathrm{O}_{2}$ por quilograma de gordura, descrito por Kaylegian \& Lindsay (1995).

Não foi observado efeito das dietas experimentais sobre TBARS (Tabela 3), apesar de o valor ter sido numericamente inferior para a dieta SojaExt+Se, nos três tempos de análise. Foi observado efeito de tempo, em que os valores de TBARS foram maiores 96 horas após a colheita do leite, quando comparado aos tempos 0 e 24 horas (Figura 2). Os resultados obtidos estão de acordo com os observados por King (1962). Esse autor correlacionou os valores de TBARS $\left(\mathrm{Abs}_{532}\right)$ com características organolépticas do leite e concluiu que valores de TBARS, entre 0,010 e 0,023 , não apresentaram "flavor" oxidado; entre 0,024 e 0,029 apresentaram "flavor" oxidado questionável; entre 0,030 e 0,040 apresentaram "flavor" oxidado leve; entre 0,041 e 0,055 , "flavor" oxidado forte, e, TBARS $>0,056$, apresentaram "flavor" oxidado muito forte.

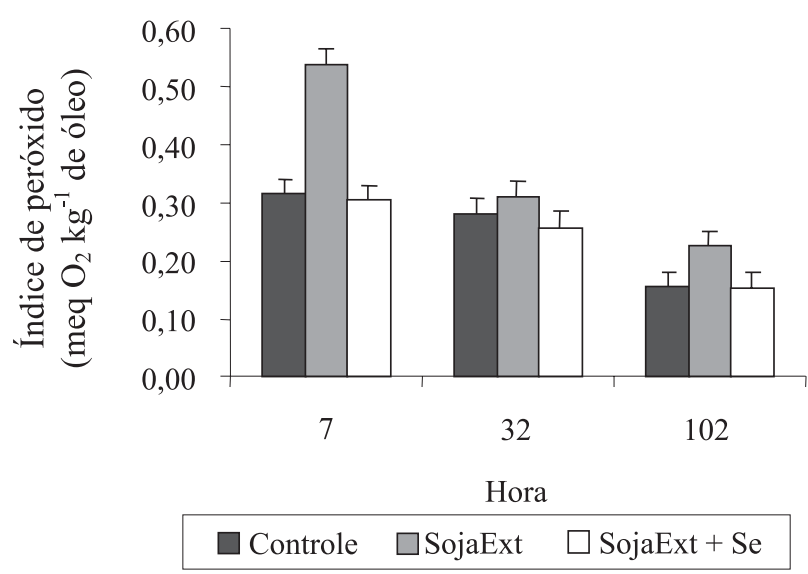

Figura 1. Índice de peróxido analisado nos tempos 7, 32 e 102 horas após a colheita do leite de vacas que receberam a dieta controle, dieta com $21 \%$ de soja extrusada (SojaExt) e dieta com $21 \%$ de soja extrusada $+5 \mathrm{mg}$ de selênio (SojaExt+Se).

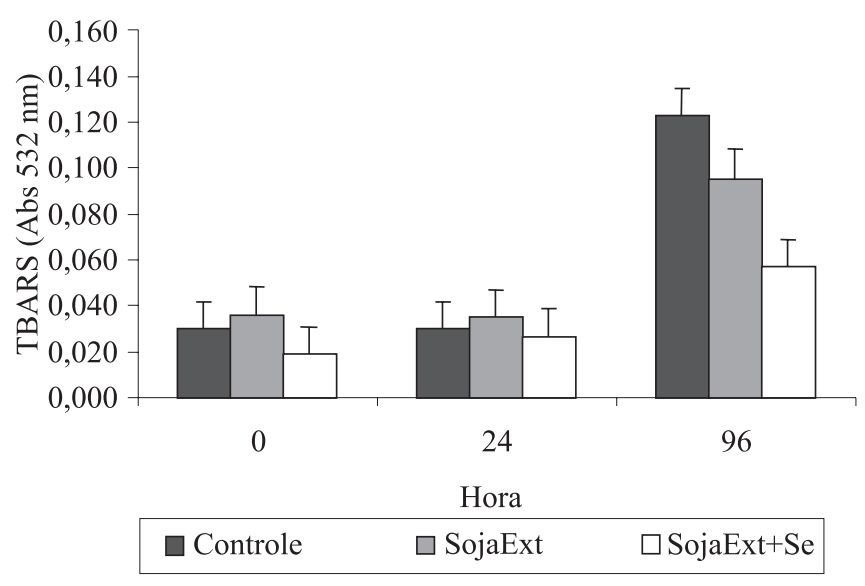

Figura 2. Análises de reações com ácido tiobarbitúrico (TBARS) 0, 24 e 96 horas após a colheita do leite de vacas que receberam a dieta controle, dieta com $21 \%$ de soja extrusada (SojaExt) e dieta com $21 \%$ de soja extrusada $+5 \mathrm{mg}$ de selênio (SojaExt+Se).

\section{Conclusões}

1. Vacas alimentadas com dieta com $21 \%$ de soja extrusada produzem leite com perfil modificado de ácidos graxos, com menor concentração de ácidos graxos saturados e maior de ácidos graxos poliinsaturados e ácido linoléico conjugado, no entanto, produzem leite mais susceptível à oxidação.

2. O enriquecimento do leite com selênio minimiza o efeito da oxidação e influencia positivamente a estabilidade do leite.

\section{Agradecimentos}

À Fapesp, pelo apoio financeiro.

\section{Referências}

ABUGHAZALEH, A.A.; SCHINGOETHE, D.J.; HIPPEN, A.R.; KALSCHEUR, K.F.; WHITLOCK, L.A. Fatty acid profiles of milk and rumen digesta from cows fed fish oil, extruded soybeans or their blend. Journal of Dairy Science, v.85, p.2266-2276, 2002.

ABUGHAZALEH, A.A.; SCHINGOETHE, D.J.; HIPPEN, A.R.; WHITLOCK, L.A. Feeding fish meal and extruded soybeans enhances the conjugated linoleic acid (CLA) content of milk. Journal of Dairy Science, v.85, p.624-631, 2002a.

ASSOCIATION OF OFFICIAL ANALYTICAL CHEMISTS. Official Methods of Analysis. 12 ${ }^{\text {th }}$ ed. Washington, 1990. 684p.

BEAM, T.M.; JENKINS, T.C.; MOATE, P.J.; KOHN, R.A.; PALMQUIST, D.L. Effects of amount and source of fat on the rates 
of lipolysis and biohydrogenation of fatty acids on ruminal contents. Journal of Dairy Science, v.83, p.2564-2573, 2000.

CHRISTIE, W.W. A simple procedure for rapid transmethylation of glycerolipids and cholesterol esters. Journal of Lipid Research, v.23, p.1072-1075, 1982.

CORL, B.A.; BAUMGARD, L.H.; DWYER, D.A.; GRIINARI, J.M.; PHILIPS, B.S.; BAUMAN, D.E. The role of delta-9-desaturase in the production of cis-9, trans-11 CLA and other delta-9 desaturated fatty acids in milk fat. Journal of Dairy Science, v.83, p.164, 2000, supplement 1 .

DHIMAN, T.R.; ANAND, G.R.; SATTER, L.D.; PARIZA, M.W. Conjugated linoleic acid content of milk from cows fed different diets. Journal of Dairy Science, v.82, p.2146-2156, 1999.

DHIMAN, T.R.; HELMINK, E.D.; McMAHON, D.J.; FIFE, R.F.; PARIZA, M.W. Conjugated linoleic acid content of milk and cheese from cows fed extruded oilseeds. Journal of Dairy Science, v.82, p.412-419, 1999a.

FENG, S.; LOCK, A.L.; GARNSWORTHY, P.C. Technical note: a rapid lipid separation method for determining fatty acid composition of milk. Journal of Dairy Science, v.87, p.3785-3788, 2004.

FONSECA, L.F.L.; SANTOS, M.V. Qualidade do leite e controle de mastite. 1.ed. São Paulo: Lemos, 2000. 175p.

GRUMMER, R.R. Effect of feed on the composition of milk fat. Journal of Dairy Science, v.74, p.3244-3257, 1991.

HARA, A.; RADIN, N.S. Lipid extraction of tissues with lowtoxicity solvent. Analytical Biochemistry, v.90, p.420-426, 1978.

INTERNATIONAL DAIRY FEDERATION. International IDF standards. $2^{\text {th }}$ ed. Brussels: IDF, 1991.

IP, C.; BANNI, S.; ANGIONI, E.; CARTA, G.; MACGINLEY, J.; THOMPSON, H.J.; BARBANO, D.; BAUMAN, D. Conjugated linoleic acid-enriched butter fat alters mammary gland morphogenesis and reduces cancer risk in rats. Journal of Nutrition, v.129, p.2135$2142,1999$.

KAYLEGIAN, K.E.; LINDSAY, R.C. Handbook of milk fat fractionation technology and application. Champaign: AOAC Press, 1995.

KEARL, L.C. Nutrients requirements of ruminants in developing countries. Logan: International Feedstuffs Institute, Utah State University, 1982. 381p.

KING, R.L. Oxidation of milk fat globule membrane material. I - Thiobarbituric acid reaction as a measure of oxidized flavor in milk and model systems. Journal of Dairy Science, v.45, p.1165-1171, 1962.

KRISTENSEN, D.; HEDEGAARD, R.V.; NIELSEN, J.H.; SKIBSTED, L.H. Oxidative stability of buttermilk as influenced by the fatty acid composition of cows' milk manipulated by diet. Journal of Dairy Research, v.71, p.46-50, 2004.

LABUZA, T.O. Oxidative changes in foods at low and intermediate moisture levels. In: DUCKWORTH, R.B. (Ed.). Food Science and Technology. New York: Academic Press, 1975. p.455-472.
LINDMARK-MANSSON, H.; AKESSON, B. Antioxidative factors in milk. British Journal of Nutrition, v.84, p.103110, 2000. (Supplement 1).

LOCK, A.L.; CORL, B.A.; BARBANO, D.M.; BAUMAN, D.E.; IP, C. The anticarcinogenic effect of trans-11 18:1 is dependent on its conversion to cis-9, trans-11 CLA by \{Delta $\} 9-$ desaturase in rats. The Journal of Nutrition, v.134, p.26982704, 2004.

MUÑIZ-NAVIERO, O.; DOMÍNGUEZ-GONZALES, R.; BERMEJO-BARRERA, A.; BERMEJO-BARRERA, P.; COCHO, J.; FRAGA, J. Study of the bioavailability of selenium in cows' milk after a supplementation of cow feed with different forms of selenium. Analytical and Bioanalytical Chemistry, v.385, p.189-196, 2006.

NICHOLSON, J.W.G.; LAURENT, A.M.; MC QUEEN, R.E.; CHARMLEY, E. The effect of feeding organically bound selenium and alpha-tocopherol to dairy cows on susceptibility of milk to oxidation. Canadian Journal of Animal Science, v.71, p.135143, 1991.

OLSON, O.E.; PALMER, L.S.; CARY, E.L. Modification of the official fluorimetric method for selenium in plants. Journal of the Association of Official Analytical Chemists, v.58, p.117-121, 1975.

PADILHA, P.C.; PINHEIRO, R.L. O papel dos alimentos funcionais na prevenção e controle do câncer de mama. Revista Brasileira de Cancerologia, v.50, p.251-260, 2004.

RAMASWAMY, N.; BAER, R.J.; SCHINGOETHE, D.J.; HIPPEN, A.R.; KASPERSON, K.M.; WHITLOCK, L.A. Composition and flavor of milk and butter from cows fed fish oil, extruded soybeans, or their combination. Journal of Dairy Science, v.84, p.21442151, 2001.

SANTOS, F.L.; SILVA, M.T.C.; LANA, R.P.; BRANDÃO, S.C.C.; VARGAS, L.H.; ABREU, L.R. Efeito da suplementação de lipídios na ração sobre a produção de ácido linoléico conjugado (CLA) e a composição da gordura do leite de vacas. Revista Brasileira de Zootecnia, v.30, p.1931-1938, 2001.

SAS INSTITUTE. Guide for personal computers. Cary: SAS Institute, 2000.

SHANTHA, N.C.; DECKER, E.A. Conjugated linoleic acid concentrations in processed cheese containing hydrogen donors, iron and dairy-based additives. Food Chemistry, v.47, p.257-261, 1993.

SILVA, D.J.; QUEIROZ, A.C.; NEVES, A.R. Análise de alimentos: métodos químicos e biológicos. 3.ed. Viçosa: Editora Universidade Federal de Viçosa, 2002.

SURAI, P.F. Selenium in nutrition and health. $1^{\text {st }}$ ed. United Kingdom: Nottingham University Press, 2006. 974p.

TIMMONS, J.S.; WEISS, W.P.; PALMQUIST, D.L.; HARPER, W.J. Relationships among dietary roasted soybeans, milk components and spontaneous oxidized flavor of milk. Journal of Dairy Science, v.84, p.2440-2449, 2001.

Recebido em 20 de junho de 2007 e aprovado em 14 de novembro de 2007 